\title{
Evaluación multicriterio de la vulnerabilidad biofísica ante inundaciones en la subcuenca río Atoyac-Oaxaca de Juárez
}

\section{Multicriteria assessment of biophysical flood vulnerability in the Atoyac-Oaxaca de Juarez subwatershed}

\author{
Marta Magdalena Chávez-Cortés*, Gilberto Binnqüist-Cervantes, Andrea Cecilia Salas-Flores \\ Universidad Autónoma Metropolitana Xochimilco. Calzada del Hueso 1100, Col. Villa Quietud, CP. 04960, Coyoacán, Ciudad \\ de México \\ *Autor de correspondencia: ccmm1320@correo.xoc.uam.mx
}

Artículo científico recibido: 01 de junio de 2015, aceptado: 22 de enero de 2016

RESUMEN. El objetivo de la investigación fue evaluar y zonificar la susceptibilidad a inundaciones de la subcuenca río Atoyac-Oaxaca de Juárez, a partir del índice de vulnerabilidad biofísica (IVBF) construido con criterios ponderados con el Proceso Jerárquico Análitico (PJA) e implementados en una plataforma de Sistema de Información Geográfica (SIG). Los resultados muestran que la vulnerabilidad clasificada como alta, ocupa $38.39 \%$ de la superficie de la cuenca. Las áreas más vulnerables son los Valles Etla, Tlacolula, Zimatlán, Ocotlán, Ejutla, Miahuatlán y la zona conurbada de la Ciudad de Oaxaca, condición que está determinada por factores fisiográficos como cercanía con cuerpos de agua, coberturas del suelo, tipos de suelo y geología. Los municipios más vulnerables son Santa Cruz Papalutla, Santa Inés Yatzeche, San Raymundo Jalpan, Asunción Ocotlán, San Antonio Castillo Velasco, Ciénega de Zimatlán y San Jacinto Amilpas. La regionalización de la vulnerbilidad biofísica ante inundaciones es una aproximación efectiva para el ordenamiento del territorio, los programas de crecimiento urbano, los atlas de riesgo y la definición de políticas de gestión ambiental.

Palabras clave: Inundaciones, Proceso Jerárquico Analítico, SIG, Vulnerabilidad, Zonificación

ABSTRACT. The objective of this work was to evaluate and zonify flood susceptibility in the Atoyac-Oaxaca de Juárez River watershed, using an index of biophysical vulnerability (BFVI) built from criteria weighted with the Analytic Hierarchy Process (AHP) and implemented on a Geographic Information System (GIS) platform. Results show that the highest vulnerability category occupies $38.89 \%$ of the watershed's area. Most vulnerable are the valleys of Etla, Tlacolula, Zimatlán, Ocotlán, Ejutla, Miahuatlán and urban neighborhoods adjacent to the city of Oaxaca, a condition determined by physiographic factors, proximity to water bodies, land cover, soil type and geology. The most vulnerable municipalities are Santa Cruz Papalutla, Santa Ynés Yatzeche, San Raymundo Jalpan, Asunción Ocotlán, San Antonio Castillo Velasco, Ciénega de Zimatlán and San Jacinto Amilpas. The zonification of biophysical vulnerability to floods is an effective approximation to land use planning, urban growth programs, risk atlases and the definition of environmental management policy.

Key words: Floods, Analytic Hierarchy Process (AHP), GIS, vulnerability, zonification

\section{INTRODUCCIÓN}

Los peligros de origen hidrometeorológico como las inundaciones, son una de las mayores amenazas para el bienestar social, debido a sus efectos multidimensionales, ya que se pierden vidas, se afecta la salud de la población por brotes de enfer- medades y se agudizan los sentimientos traumáticos en la población afectada (Jha et al. 2012, Dewan 2013). También se altera la estructura y funcionamiento de los sistemas ecológicos del paisaje, se degradan los recursos que sustentan las actividades económicas, se pierde o daña la infraestructura y los servicios, y se pone en riesgo la seguri- 
dad física y patrimonial de las personas. Las consecuencias extremas de estos fenómenos pueden redirigir el carácter de las instituciones sociales, además de resultar en la aparición, permanente y costosa, de nuevas regulaciones para las generaciones futuras, y perturbar la estabilidad social (Fordham et al. 2013). Una muestra de la dimensión de estos impactos negativos es las pérdidas económicas que provocaron las inundaciones en México de 1980 a 1990, que fueron de 4537 millones de dólares (CENAPRED 2014). Esta situación se torna seria al considerar las variaciones climáticas, ya que bajo este escenario los países emergentes como México serán afectados por sus altos índices de población y pobreza (Adger 2006).

En este contexto, el estado de Oaxaca es de particular interés, ya que su ubicación geográfica y condiciones climáticas lo hacen propenso a embate de huracanes (Rodríguez 2011). Además, de ríos caudalosos de respuesta rápida que causan inundaciones (CIEDD 2014). Aunado a esto, el territorio de la subcuenca ha sufrido modificaciones, siendo el cambio de uso de suelo más importante, el de vivienda (Binnqüist 2011). Por ello, ante el posible incremento en la frecuencia e intensidad de futuros eventos hidrometeorológicos, causados por el cambio climático, es importante realizar la gestión integral del territorio, con acciones preventivas, de mitigación y adaptación (CMM 2013). Por lo que los estudios de vulnerabilidad son importantes para identificar las características internas que hacen que un sistema sea proclive a los efectos adversos de un evento natural (Arreguín et al. 2011).

Las inundaciones están relacionadas con la ocurrencia de fenómenos hidrometeorológicos (Dewan 2013). La probabilidad de que una inundación se convierta en desastre, se debe a la ocurrencia simultánea del peligro y la vulnerabilidad (Becker 2014). La vulnerabilidad es la condición que define la predisposición intrínseca de un sistema a ser afectado por un fenómeno; mientras que el peligro se asocia con una serie de eventos naturales o antrópicos, que tienen el potencial de causar daño (Cardona 2004). El análisis de vulnerabilidad es la piedra angular de la investigación de riesgos, sobre todo en los fenómenos hidrometeorológicos (Fekete et al. 2009, Dewan 2013). Lo que, se debe a que este enfoque, investiga los peligros y las causas que dan lugar a condiciones de vulnerabilidad, las cuales pueden ser de orden biofísico, social, económico, político y cultural (Adger 2006, Dewan 2013). La vulnerabilidad biofísica es aquella que se refiere a la fragilidad de un socioecosistema ante un fenómeno (Cardona 2004). La vulnerabilidad a las inundaciones, está determinada por el tipo y uso de suelo, cobertura, grado de inclinación de la pendiente, distancia a los ríos, geología y geoformas (López y Sánchez 2011, Balica et al. 2012). No obstante que la vulnerabilidad a fenómenos naturales es un tema en discusión (Fordham et al. 2013), las evaluaciones de la vulnerabilidad pueden contribuir en la reducción de los desastres naturales (Gao et al. 2007), y promueven el desarrollo de una sociedad resiliente a los desastres (Birkmann 2006, Becker 2014). Además de que ayudan a desarrollar medidas de mitigación, permiten identificar factores de vulnerabilidad (Dewan 2013), y constituyen la base para la toma de decisiones en la planificación y gestión territorial (Zamorano y Toscana 2013).

Se tienen distintos marcos de referencia para la cualificación y evaluación de la vulnerabilidad. De los más empleados están el BogardiBirkman-Cardona (BBC), el de Cebolla, el de Presión y Relajación (PAR) y la Evaluación Integral de Amenazas Múltiples (IEMT) (Birkman 2007, Dewan 2013). Todos parten de nociones diferentes de vulnerabilidad, con enfoques distintos, pero coinciden en que la vulnerabilidad es un tema que debe abordarse con un análisis de criterios múltiples (ACM) (Eakin y Bojórquez 2008, Fordham et al. 2013). Sin embargo, no consideración el componente espacial de la vulnerabilidad, importante en las inundaciones, ya que los fenómenos que las detonan, así como las condiciones que determinan sus impactos toman lugar en el espacio geográfico (Dewan 2013). Los avances recientes de la tecnología de SIG, permiten la integración de datos cualitativos y cuantitativos en un ACM (Llorente et al. 2009). Entre los trabajos que abordan este tipo de enfoque cuali-cuantitativo y espacial se encuen- 
tran Dang et al. (2011) la evaluación de parámetros de riesgo a inundaciones en Vietnam; mientras que Gao et al. (2007) lo utilizan para evaluar inundaciones en China, en tanto que Meyer et al. (2009) y Sebastian et al. (2011) lo emplean con el mismo propósito en Alemania. También Sidayyo et al. (2014) aplicaron el Proceso Jerárquico Analítico (PJA) para evaluar el riesgo de inundación. Por otro lado Mendoza et al. (2014) reportan el uso del análisis de vulnerabilidad biofísica para el estudio de riesgos de inundación en Toluca, y Sánchez y Martínez (2012) en Venezuela. Los índices de vulnerabilidad y su cartografía son herramientas valiosas para la toma de decisiones en la planificación del uso de suelo, y en el establecimiento de zonas de protección y conservación necesarias para mantener bienes y servicios ambientales (Dewan 2013, Torres et al. 2014). Por lo anterior el objetivo del estudio fue evaluar y zonificar la susceptibilidad a inundaciones de la subcuenca río Atoyac-Oaxaca de Juárez, mediante la construcción y aplicación de un índice de vulnerabilidad biofísica (IVBF).

\section{MATERIALES Y MÉTODOS}

El presente estudio se realizó en la subcuenca del río Atoyac-Oaxaca de Juárez, con superficie de $3727 \mathrm{~km}^{2}$, localizada al sureste de la República Mexicana en la porción centro del estado de Oaxaca, región hidrológica 20 Costa Chica-río Verde, en la cuenca del río Atoyac (Figura 1). La subcuenca comprende los distritos de Etla, Ixtlán, Centro, Tlacolula, Ocotlán, Ejutla y parte de Miahuatlán y Sola de Vega, tiene 1003 localidades con una población de 1012492 habitantes y densidad poblacional de 172.68 habitantes por $\mathrm{km}^{2}$ (INSO 2014).

El trabajo se realizó con enfoque multicriterios, el juicio de expertos y SIG. El procedimiento metodológico comprendió tres fases. En la primera se realizó la caracterización del área de estudio con base en la selección de los criterios de grado de inclinación de las pendientes, cobertura y uso de suelo, distancia a ríos y cuerpos de agua, tipos de suelo y sustratos geológicos (López y Sánchez 2011, Balica et al. 2012). Además de cartografía digital para constituir la geobase de datos, que se complementó con los mapas de distancia a cuerpos de agua y pendientes.

En la segunda fase se construyó el IVBF por medio del Proceso Jerárquico Analítico (Saaty 1990), utilizando como criterios de la jerarquía la inclinación de la pendiente, cobertura y uso de suelo, distancia a ríos y cuerpos de agua, tipos de suelo y geología. Cada uno de estos criterios se dividió en distintas clases, las cuales constituyeron los indicadores de la variación espacial de dichos criterios. La inclinación de la pendiente se dividió de 0 a $6^{\circ}, 6^{\circ}$ a $15^{\circ}, 15^{\circ}$ a $25^{\circ}$ y $25^{\circ}$ a $80^{\circ}$. Mientras que la cobertura y uso de suelo se dividió en las clases de cuerpos de agua, bosque/selva, matorral/pastizal, cultivos y zona urbana. En tanto que la distancia a ríos y otros cuerpos de agua se separó en las categorías de 0 a $478.19 \mathrm{~m}, 478.19$ a $996.24 \mathrm{~m}, 996.24$ a 1574.06 m, 1574.06 a 2371.05 m y 2371.05 a 5080.83 $\mathrm{m}$. Los tipos de suelo se dividieron en las categorías vertisol/phaeozem, acrisol, luvisol/cambisol y leptosol/regosol. En tanto que la geología se separó en A (gneis, granito), B (aluvial, residual, lacustre), C (lutita, andesita, basalto, lutita-arenisca, calizas, caliza-lutita), D (limolita-arenisca,conglomerado) y E (arenisca, tobacida, monzonita).

Se establecieron los pesos relativos a cada uno de los criterios e indicadores construyendo matrices de comparación por pares de cada nivel de la jerarquía, utilizando el juicio de expertos para determinar la importancia relativa de los criterios e indicadores para favorecer las inundaciones. Para cada matriz, se calculó el índice de consistencia interno, que debe ser menor a 0.1 para ser aceptable. Una vez que la consistencia se validó para cada matriz, se obtuvo el factor de peso de cada criterio e indicador de los distintos niveles de jerarquía, calculando el eigenvector de cada matriz. El valor del IVBF se calculó mediante la siguiente fórmula:

$$
I V B F=\sum_{i=1}^{n} \sum_{j=1}^{m} W_{i} W_{i j}
$$

Donde IVBF es el índice de vulnerabilidad 


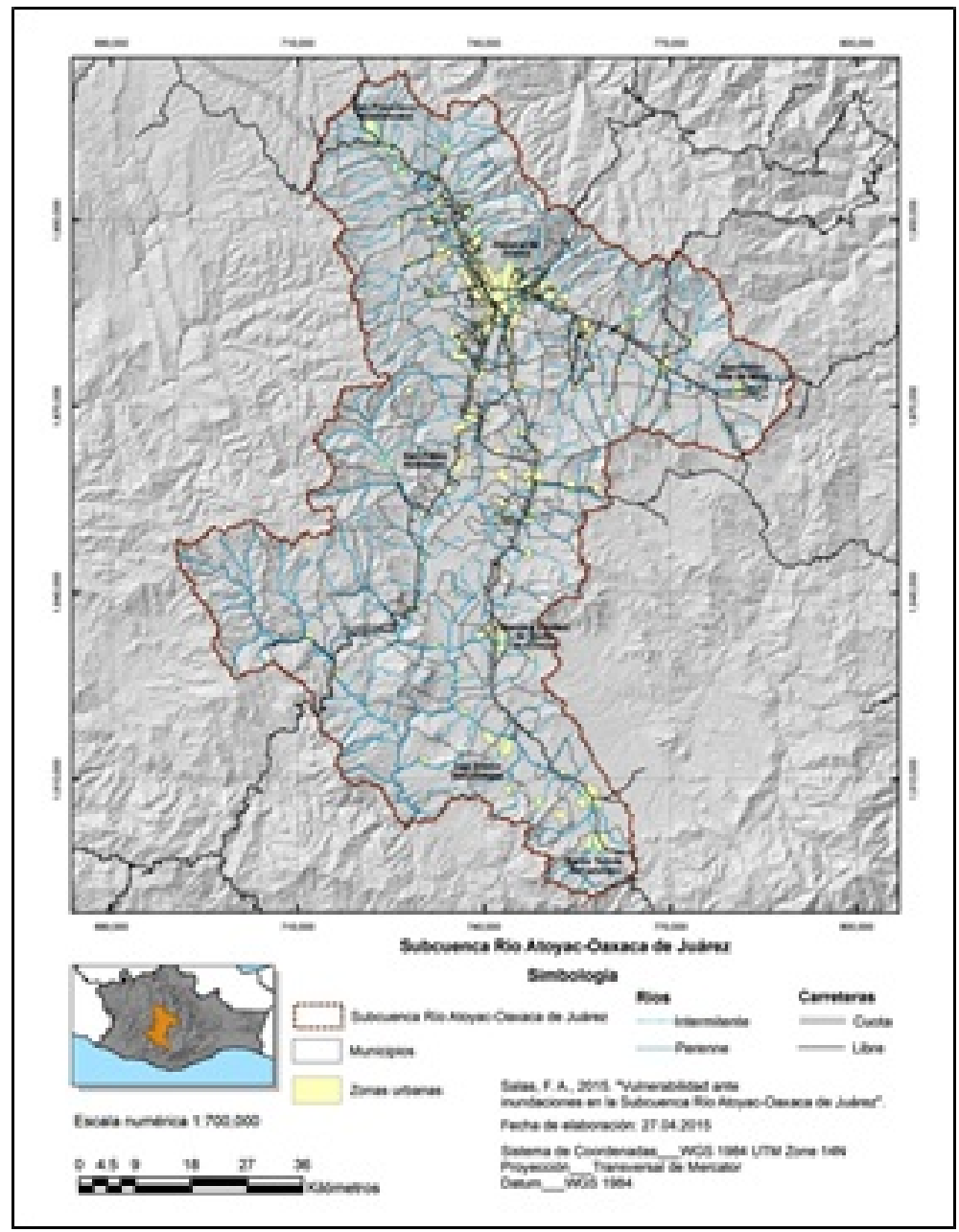

Figura 1. Ubicación geográfica de la subcuenca Rio Atoyac-Oaxaca de Juárez.

biofísica, $W_{i}$ es el peso relativo del i-ésimo criterio y $W_{i j}$ es el valor de ponderación del j-ésimo indicador considerado dentro del i-ésimo criterio (Saaty 1990).

Este proceso de agregación matemática se articuló en la plataforma SIG (ArcView 10.1) con álgebra de mapas, para obtener los mapas de vulnerabilidad explicada pora cada criterio, así como el del IVBF. Para facilitar la comprensión de la información espacial que proporciona el mapa de vulnerabilidad biofísica, se clasificó en las categorías de alta, moderada y baja, asignando un código de colores de rojo para la categoría alta, amarillo para la moderada y verde para la baja.
En la tercera etapa se realizó la recopilación histórica de la ocurrencia de fenómenos hidrometeorológicos que han ocasionado inundaciones en los últimos 27 años en el área de estudio (Tabla 1), a partir de la cual se realizó la comparación de incidencia de estos fenómenos en los municipios que calificaron en la categoría de alta vulnerabilidad ante inundaciones, para validar la zonificación elaborada. 
Tabla 1. Principales fenómenos hidrometeorológicos que han originado inundaciones en la subcuenca Río Atoyac- Oaxaca de Juárez.

\begin{tabular}{|c|c|c|}
\hline Año & Nombre & $\begin{array}{l}\text { Precipitación máxima } \\
\text { registrada en } 24 \mathrm{~h}(\mathrm{~mm})\end{array}$ \\
\hline 1988 & Gilbert & 505 \\
\hline 1991 & Depresión Tropical DT 5E & 70 \\
\hline 1993 & Beatriz & 122.5 \\
\hline 1996 & Cristina & 150 \\
\hline \multirow[t]{3}{*}{1997} & Pauline & 411 \\
\hline & Olaf & 110 \\
\hline & Rick & 150 \\
\hline 1999 & Greg & 200 \\
\hline \multirow[t]{2}{*}{2000} & Rosa & 103 \\
\hline & Carlota & 310 \\
\hline 2001 & Iris & 122 \\
\hline 2002 & Isidoro & 300 \\
\hline 2003 & Carlos & 110 \\
\hline \multirow[t]{2}{*}{2005} & Stan & 273 \\
\hline & Emily & 251 \\
\hline \multirow[t]{3}{*}{2010} & Depresión tropical $11 e$ & 121.5 \\
\hline & Karl & 127.5 \\
\hline & Matthew & 182 \\
\hline \multirow[t]{4}{*}{2011} & Beatriz & 222.5 \\
\hline & Depresión tropical D-12 & 79 \\
\hline & Arlene & 231.5 \\
\hline & Harvey & 172.5 \\
\hline 2012 & Carlota & 350 \\
\hline \multirow[t]{2}{*}{2013} & Manuel & 505 \\
\hline & Ingrid & 348 \\
\hline 2014 & Trudy & 250 \\
\hline
\end{tabular}

\section{RESULTADOS}

\section{Criterios e indicadores de vulnerabilidad biofísica ante inundaciones}

La opinión de expertos, permitió identificar los criterios, indicadores y pesos relativos, que influyen en la vulnerabilidad biofísica a las inundaciones de la subcuenca del río Atoyac-Oaxaca de Juárez. Los índices de consistencia medidos en las matrices de comparación indican que las bases del juicio de expertos son consistente. La pendiente del terreno, distancia a ríos y cuerpos de agua, cobertura y tipo de suelo, son los factores de mayor relevancia para las inundaciones ante un fenómeno hidrometeorológico en la subcuenca (Tabla 2). Para la pendiente, el juicio de expertos indica que cuando el terreno tiene entre 0 y $6^{\circ}$ de inclinación, es más susceptible a inundarse, mientras que pendientes superiores de $25^{\circ}$ no representan condición de suceptibilidad.

La unidad de suelo vertisol/phaozem facilita casi dos veces más las inundaciones que el acrisol y, éste último, tres y seis veces más que los luvisoles/cambisoles y los leptosoles/regosoles. Los juicios de valor indican que los dos primeros rangos de la distancia a cuerpos de agua son los que más favorecen la vulnerabilidad biofísica, por lo que los primeros $400 \mathrm{~m}$ a una fuente de agua son críticos en la subcuenca para que ocurra una inundación. Para la cobertura de suelo, la zona urbana es la que más peso tiene para favorecer las inundaciones. A éstas le siguen las áreas cultivadas con casi la mitad del peso que la urbana y las de matorral/pastizal con tres veces menos que la zona urbana. Mientras que el bosque/selva es la cobertura vegetal que favorece menos las inundaciones.

\section{La zonificación de la vulnerabilidad biofísica en la subcuenca}

La evidencia histórica sobre peligros hidrometeorológicos e inundaciones confirma los resultados obtenidos en el mapa del IVBF. El análisis espacial indicó la presencia de áreas de alta vulnerabilidad a lo largo de los ríos Atoyac y el Salado, 
Tabla 2. Criterios de vulnerabilidad biofísica y sus pesos relativos.

\begin{tabular}{|c|c|c|c|}
\hline Criterios & Peso relativo & Indicador & Peso relativo \\
\hline \multirow[t]{4}{*}{ Pendiente de terreno } & 0.43 & 0 a $6^{\circ}$ & 0.55 \\
\hline & & 6 a $15^{\circ}$ & 0.25 \\
\hline & & 15 a $25^{\circ}$ & 0.16 \\
\hline & & 25 a $80^{\circ}$ & 0.04 \\
\hline \multirow[t]{5}{*}{ Cobertura y uso de suelo } & 0.16 & Cuerpos de agua & 0.00 \\
\hline & & Bosque/Selva & 0.06 \\
\hline & & Matorral/Pastizal & 0.13 \\
\hline & & Cultivos & 0.29 \\
\hline & & Zona urbana & 0.52 \\
\hline \multirow{5}{*}{$\begin{array}{l}\text { Distancia a ríos y } \\
\text { cuerpos de agua }\end{array}$} & 0.26 & 0 a $478 m$ & 0.48 \\
\hline & & 478 a $996 m$ & 0.28 \\
\hline & & 996 a $1574 m$ & 0.12 \\
\hline & & 1574 a $2371 m$ & 0.07 \\
\hline & & 2371 a $5081 \mathrm{~m}$ & 0.04 \\
\hline \multirow[t]{4}{*}{ Tipos de suelo } & 0.10 & Vertisol/Phaeozem & 0.61 \\
\hline & & Acrisol & 0.26 \\
\hline & & Luvisol/Cambisol & 0.09 \\
\hline & & Leptosol/Regosol & 0.04 \\
\hline \multirow[t]{5}{*}{ Geología } & 0.06 & A; Gneis, granito & 0.50 \\
\hline & & B; Aluvial, residual, lacustre & 0.26 \\
\hline & & C; Lutita, andesita, basalto, lutita-arenisca, calizas, caliza-lutita & 0.13 \\
\hline & & D; limolita-arenisca, conglomerado & 0.07 \\
\hline & & $\mathrm{E} ;$ arenisca, tobacida, monzonita & 0.03 \\
\hline
\end{tabular}

Tabla 3. Condiciones de pobreza, marginalidad y desarrollo humano en los municipios con alta vulnerabilidad biofísica.

\begin{tabular}{llllllll}
\hline $\begin{array}{l}\text { Municipios con 100 \% de su superfi- } \\
\text { cie en alta vulnerabilidad ante inun- } \\
\text { daciones }\end{array}$ & $\begin{array}{l}\text { Índice } \\
\text { rezago } \\
\text { social }\end{array}$ & $\begin{array}{l}\text { de } \\
\text { San Jacinto Amilpas }\end{array}$ & $\begin{array}{l}\text { Grado } \\
\text { rezago } \\
\text { social }\end{array}$ & $\begin{array}{l}\text { de } \\
\text { marginación }\end{array}$ & $\begin{array}{l}\text { Grado de } \\
\text { marginación }\end{array}$ & $\begin{array}{l}\text { Índice } \\
\text { desarrollo } \\
\text { Humano }\end{array}$ & $\begin{array}{l}\text { derado } \\
\text { Desarrollo } \\
\text { Humano }\end{array}$ \\
\hline Guadalupe Etla & -1.29 & Muy bajo & -1.75858 & Muy bajo & 0.8862 & $\begin{array}{l}\text { Alto } \\
\text { Bedio Alto }\end{array}$ \\
Ciénega de Zimatlán & -0.87 & Muy bajo & -0.95606 & Bajo & 0.8331 & Medio \\
San Raymundo Jalpan & -0.51 & Bajo & -0.43901 & Medio & 0.8139 & Medio Alto \\
Santa Cruz Papalutla & -0.38 & Bajo & -0.20894 & Medio & 0.8238 & Medio Alto \\
San Pedro Apóstol & 0.28 & Medio & 0.20394 & Medio & 0.7438 & Medio Alto \\
San Antonino Castillo Velasco & 0.64 & Medio & 0.60213 & Alto & 0.7656 & Medio Bajo \\
Santiago Matatlán & 0.89 & Alto & 0.70662 & Alto & 0.7623 & Medio Alto \\
Santa Lucía Ocotlán & 1.06 & Alto & 0.99170 & Muy alto & 0.7112 & Medio Bajo \\
Santa Inés Yatzeche & 1.22 & Alto & 1.00743 & Muy alto & 0.6904 & Medio Bajo \\
Santiago Apóstol & 1.41 & Alto & 1.25726 & Muy alto & 0.6727 & Medio Bajo \\
Asunción Ocotlán & 1.72 & Alto & 1.33836 & Muy alto & 0.7056 & Medio Alto \\
\hline
\end{tabular}

en los Valles de Etla, Tlacolula, Zimatlán Ocotlán, Ejutla, Miahuatlán, y en la ciudad de Oaxaca de Juárez (Figura 2). Mientras que las áreas de menor vulnerabilidad se distribuyeron alrededor de las áreas de alta vulnerabilidad. En tanto que las áreas de vulnerabilidad media presentan un patrón más disperso y siguen los cauces de segundo orden.

Las áreas de alta vulnerabilidad representan $38.39 \%$ de la superficie de la subcuenca (225 664 ha), mientras que las de mediana vulnerabilidad constituyen $35.28 \%$ (207 $408 \mathrm{ha}$ ) y las de baja vulnerabilidad $26.32 \%$ (154 736 ha). En conjunto, las áreas de alta vulnerabilidad agrupan $57 \mathrm{mu}$ nicipios, pero destacan Asunción Ocotlán, Ciénega de Zimatlán, Guadalupe Etla, Santa Cruz Papalutla, San Antonino Castillo Velasco, San Jacinto Amilpas, San Pedro Apóstol, Santiago Apóstol, Santiago Matatlán, San Raymundo Jalpan, Santa Inés Yatzeche y Santa Lucía Ocotlán, con el total de su territorio en alta vulnerabilidad. De los 57 municipios de alta vulnerabilidad, el $36.84 \%$ tienen un índice de desarrollo humano alto, mientras que $22.81 \%$ un índice bajo. En rezago social, es dominante el rezago medio en el $38.6 \%$ de los municipios 


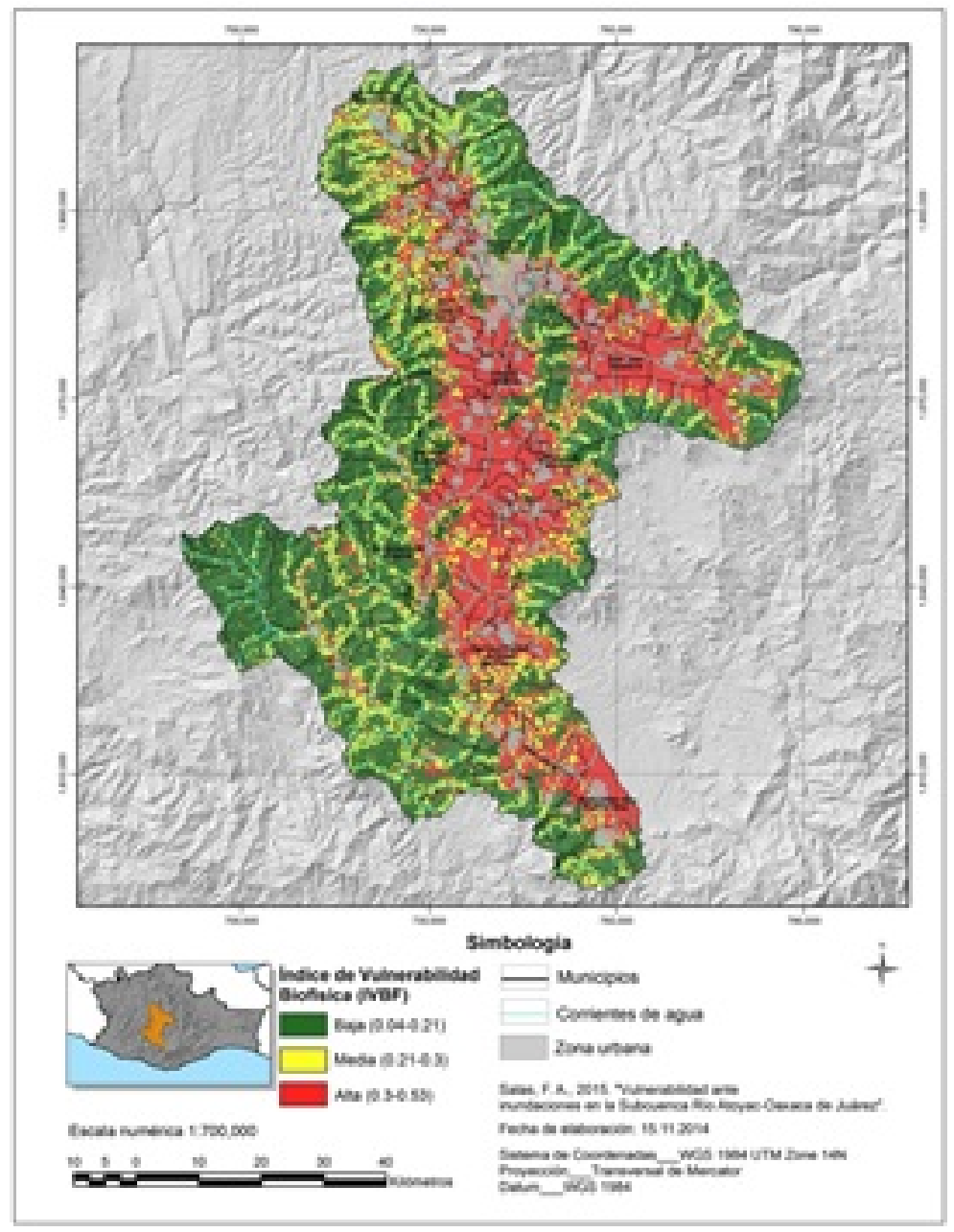

Figura 2. Ubicación geográfica de la subcuenca Rio Atoyac-Oaxaca de Juárez.

de alta vulnerabilidad, seguido por un rezago bajo en el $21.05 \%$. El grado de marginación de estos municipios es medio en $30.15 \%$ de ellos, mientras que el $27.94 \%$ tiene muy alta marginación. Por lo que no siempre la vulnerabilidad natural se asocia con condiciones de alta vulnerabilidad social - económica. En la Tabla 3 destaca el municipio de San Jacinto Amilpas que tiene baja vulnerabilidad socioeconómica, pero con inundaciones recurrentes. Lo que lleva a pensar en aspectos como la urbanización y la infraestructura urbana como factores que pueden explicar en mayor medida su estatus.
Índice de vulnerabilidad biofísica (IVBF) y relación con criterios biofísicos

Los valores del IVBF oscilaron entre 0.04 y 0.53 . Siguiendo el algoritmo de cortes naturales, la diferencia entre estos dos valores se dividió en tres rangos para dar lugar a las categorías de vulnerabilidad alta para el rango de 0.3 a 0.53 , media de 0.21 a 0.3 y baja de 0.04 a 0.21 .

Las áreas más vulnerables a inundaciones son las unidades agrícolas en las planicies, llanuras en pie de montes y lomeríos o terrazas, con pendientes menores de $12^{\circ}$ de inclinación, cercanas a cuerpos o corrientes de agua que pueden desbordarse. Estas unidades se encuentran en los suelos vertisol, 
phaeozem y acrisol, en una unidad hidrogeológica constituida por rocas tipo gneis o granito, aluvial, residual y lacustre. Las áreas medianamente vulnerables son los matorrales y pastizales ubicados en los pie de monte, con pendientes entre $12^{\circ} \mathrm{y}$ $22^{\circ}$, alejadas de cuerpos o corrientes de agua. Estas unidades se encuentran ubicadas en suelos luvisoles o cambisoles, la unidad hidrogeológica sobre la que están constituidas son las rocas de tipo lutita, andesita, basalto, caliza, limolita y conglomerado. Las áreas menos vulnerables corresponden a bosques y selvas ubicadas en las sierras con pendientes mayores a $22^{\circ}$, alejadas de cuerpos o corrientes de agua. Estas unidades están situadas en suelos leptosoles o regosoles sobre unidades hidrogeológicas formadas por rocas de tipo arenisca, tobacida o monzonita.

Los valores de los indicadores que conforman cada criterio se distribuyen de forma heterogénea, por lo que al sobreponerse, se generan gradientes de condiciones que favorecen las inundaciones. La influencia relativa de todos estos criterios se puede observar en los patrones espaciales de la vulnerabilidad biofísica (Figura 2). Pero también en la cantidad de área sobre la que influyen, dentro del gradiente. Por ello, al analizar de forma cuantitativa la distribución espacial de las coberturas y usos de suelo con respecto a la categoría de alta vulnerabilidad (Tabla 4), se aprecia que la mayoría de la superficie altamente proclive a las inundaciones tiene uso de suelo agrícola $(22.93 \%)$ y en segundo lugar el uso urbano (7.52 \%). En contraste, las áreas de baja vulnerabilidad están cubiertas por bosques y selvas ( 30.67 $\%)$ en primer lugar y por pastizales en segundo lugar $(8.06 \%)$.

Los resultados indican que los suelos que subyacen en las áreas de alta susceptibilidad a inundaciones son en primer lugar el vertisol/phaeozem (16.24\%), y en segundo lugar el leptosol/regosol con $14.69 \%$ (Tabla 4). Mientras que la mayor parte de la superficie ocupada por la categoría de baja vulnerabilidad (34.09\%) es de tipo leptosol y regosol, y $8.79 \%$ de luvisol/cambisol. En tanto que la unidad B (aluvial, residual y lacustre) y la unidad A (gneis-granito), son las rocas que subyacen en 8.51 y $15.81 \%$ de la superficie que ocupa la categoría de alta vulnerabilidad. Mientras que $19.23 \%$ de la superficie de baja vulnerabilidad se encuentra ubicada sobre la unidad C (Lutita, andesita, basalto, lutita-arenisca, calizas y caliza-lutita), y $14.55 \%$ sobre la unidad A (gneis, granito).

\section{DISCUSIÓN}

Las áreas de alta vulnerabilidad están asociadas con las llanuras y planicies. Debido a que un relieve suave y poco accidentado favorece el avance de los excedentes de agua, lo que aumenta la posibilidad de inundar amplias zonas (Dewan 2013, Siddayo et al. 2014). Los tipos de suelo vertisol y phaozem, que en dichas áreas, facilitan las inundaciones debido a sus características de baja permeabilidad, lo que incrementa la lámina de escorrentía superficial (Zapata-Sierra y Manzano-Agugliaro 2008). Para estas áreas, los primeros $400 \mathrm{~m}$ de los cuerpos de agua son críticos para inundación, lo que debe alertar a los planificadores del territorio, ya que la subcuenca del río Atoyac-Oaxaca de Juárez tiene un fuerte proceso de urbanización en sus zonas planas aledañas a los cuerpos de agua, lo que impacta de forma negativa sobre el control de avenidas de ríos (Montoya et al. 2009), e incrementa los efectos negativos de inundación sobre los asentamientos humanos (Zamorano y Toscana 2013).

Las áreas de menor vulnerabilidad a sufrir inundaciones tienen distancias mayores a 2300 $\mathrm{m}$ de los cuerpos de agua, correspondiendo a las sierras con sus pendientes pronunciadas. El hecho de que las áreas de menor vulnerabilidad estén asociadas con pendientes mayores a $25^{\circ}$ se debe a que las pendientes actúan como superficies dispersoras de escurrimientos, por su baja capacidad de acumular agua (Palacio et al. 2005). A esto se añade el hecho de que estas áreas se asientan sobre unidades formadas por rocas de tipo limolitaarenisca, conglomerados, arenisca, tobacida y monzonita, que tienen alta permeabilidad (Fetter 2001), lo que limita la acumulación de agua (Zamorano y Toscana 2013). 
Tabla 4. Distribución porcentual de las unidades geológicas, edáficas, y coberturas y usos del suelo con respecto a la vulnerabilidad biofísica en la subcuenca.

\begin{tabular}{lrrrr}
\hline Criterios y atributos biofísicos & Alta & Media & Baja & \\
Unidades geológicas & Superficie \% & Superficie \% & Superficie \% & Total \\
A. Gneis, granito & & & & \\
B. Aluvial, residual y lacustre & 8.51 & 9.19 & 14.55 & 32.25 \\
C. Lutita, andesita, basalto, lutita-arenisca, & 15.81 & 0.59 & 0.3 & 16.7 \\
calizas y caliza-lutita & 6.91 & 8.74 & 19.23 & 34.88 \\
D. Limolita-arenisca y conglomerado & & & & \\
E. Arenisca, tobacida y monzonita & 1.52 & 1.06 & 1 & 3.58 \\
Total & 1.28 & 1.87 & 9.71 & 12.86 \\
Unidades edáficas & 34.03 & 21.45 & 44.79 & 100.00 \\
Vertisol/Phaeozem & & & & \\
Acrisol & 16.24 & 2.5 & 1.23 & 19.97 \\
Luvisol/Cambisol & 0.05 & 0.35 & 0.69 & 1.09 \\
Leptosol/Regosol & 3.04 & 2.56 & 8.79 & 14.39 \\
Total & 14.69 & 15.77 & 34.09 & 64.55 \\
Tipos de coberturas o usos de suelo & 34.02 & 21.18 & 44.8 & 100.00 \\
Agricultura & & & & \\
Bosque & 22.93 & 5.99 & 1.73 & 30.65 \\
Pastizal & 0.98 & 8.33 & 30.67 & 39.98 \\
Matorral & 4.33 & 6.97 & 8.06 & 19.36 \\
Selva & 0.12 & 0 & 0 & 0.12 \\
Zona urbana & 0.05 & 0.31 & 0.80 & 1.16 \\
Total & 7.52 & 0.83 & 0.15 & 8.5 \\
\hline Fuente: CONAPO (2010), CONEVAL (2010) y PNUD & 35.93 & 22.43 & 41.41 & 100.00 \\
\hline
\end{tabular}

Las áreas de mediana vulnerabilidad están asociadas fundamentalmente a los pies de monte, ya que sus características morfológicas hacen de estos espacios zonas de tránsito fluvial, en donde no se favorece la permanencia del agua (Zamorano y Toscana 2013). Estas áreas están formadas por unidades de rocas de tipo lutita, andesita, basalto, lutita-arenisca, calizas y caliza-lutita, que tienen una permeabilidad moderada (Fetter 2001). Los bosques y selvas son determinantes en la disminución de la vulnerabilidad, por lo que las áreas menos vulnerables de la subcuenca se localizan en estos tipos de cobertura vegetal (Dewan 2013, Zamorano y Toscana 213). Ya que la vegetación ejerce regulación hidrológica, debido a que modifica la infiltración por interceptar de forma parcial el agua de lluvia, modifica la intensidad y el diámetro de gota del agua que cae al suelo, lo que reduce la intensidad de la lluvia y aumenta la cobertura de las gotas, para permitir mayor infiltración del agua. (Chapin III et al. 2002). Además de que las raíces o sus restos generan macroporos, por los que el agua circula a mayor velocidad (Jarrett y Hoover 1985, Archer et al. 2002). Por lo que el mantenimiento y preservación de los bosques y selvas juegua un papel primordial como mecanismo regulador de inundaciones (Cotler 2004).

Mantener los bosques y selvas presentes la subcuenca del río Atoyac-Oaxaca de Juárez tiene importancia en el contexto del estado de la cobertura vegetal, ya que la disminución de la vegetación primaria es indicio de un proceso de deforestación. De acuerdo con Cuevas et al. (2010) entre el 25 y $50 \%$ del territorio de esta subcuenca ha sido alterado, debido a que las coberturas originales del suelo han sido sustituidas por usos de suelo de carácter antrópico como la urbanización informal en los lomeríos (Binnqüist 2011); lo que tiene importantes efectos en su funcionalidad hídrica. Evidencia de estos efectos se encuentra en el estudio de Villarreal et al. (2011), quienes relacionaron el cambio de uso de suelo en esta subcuenca de 1990 a 2005, con el aumento en la lámina de escurrimiento. La cobertura agrícola aumentó de 47.84 a $53.74 \%$, mientras que el área urbana pasó de $7.37 \%$ a $14.22 \%$ y las áreas de pastizal disminuyeron de 3.77 a $2.25 \%$. Al mismo tiempo, la variación del tirante de la lámina de escurrimiento de 1990 a 2005 aumentó 3 mm, 
lo que significa mayor aporte de agua a los cauces y también la posibilidad de un mayor arrastre de sedimentos, lo que influye en las inundaciones.

\section{CONCLUSIONES}

El IVBF es una herramienta pertinente y coherente para regionalizar la subcuenca río AtoyacOaxaca de Juárez, los 57 municipios que tienen entre 50 y $100 \%$ de su superficie en categoría de alto riesgo fueron declarados en emergencia o zona de desastre natural alguna vez. De los criterios usados para construir el IVBF, la pendiente del terreno y las geoformas son equivalentes en términos de su aporte de información. La zonificación del territorio mostró que los municipios más vulnerables son Asunción Ocotlán, Ciénega de Zimatlán, Guadalupe
Etla, Santa Cruz Papalutla, San Antonino Castillo Velasco, San Jacinto Amilpas, San Pedro Apóstol, Santiago Apóstol, Santiago Matatlán, San Raymundo Jalpan, Santa Inés Yatzeche y Santa Lucía Ocotlán; por lo que son focos de atención prioritaria para la prevención de desastres ante las variaciones climáticas. La integración de los resultados del IVBF dentro del ambiente del SIG, fue adecuado para regionalizar la susceptibilidad a inundaciones y relacionarla con las condiciones de pobreza y bienestar social presentes en la subcuenca río Atoyac-Oaxaca de Juárez.

\section{AGRADECIMIENTOS}

A los revisores por las aportaciones para mejorar el artículo.

\section{LITERATURA CITADA}

Adger W (2006) Vulnerability. Global Environmental Change 16: 268-281.

Archer NAL, Quinton JN, Hess TM (2002) Below ground relationships of soil texture, roots and hydraulic conductivity in two-phase mosaic vegetation in South-east Spain. Journal of Arid Environment 52: 535-553.

Arreguín CF, Rosengaus M, Acosta A, Chávez R, López M, Hungsberg U, et al. (2009) Manual para el control de las inundaciones. Comisión Nacional del Agua. México. 326p.

Balica SF, Wright NG, Meulen FV (2012) A flood vulnerability index for coastal cities and its use in assessing climate change impacts. Journal of the International Society for the Prevention and Mitigation of Natural Hazards 52: 1-35.

Becker P (2014) Sustainability Science. Managing risk and resilience for sustainable development. 1a Edición. Elsevier. Amsterdam, The Netherlands. 302p.

Binnqüist G (2011) Actualización del plan de ordenamiento de la zona conurbada de la ciudad de Oaxaca, fase I. Secretaría de las Infraestructuras y Ordenamiento Territorial Sustentable del estado de Oaxaca, Universidad Autónoma Metropolitana, Unidad Xochimilco, Fondo Metropolitano. México. 560p.

Birkmann J (2007) Risk and vulnerability indicators at different scales: Applicability, usefulness and policy implications. Environmental Hazards 7: 20-31.

CMM (2013) Ciudades con Iniciativas Climáticas: Oaxaca. Centro Mario Molina. México. 6 p http://centro mariomolina.org Fecha de consulta 2 de abril del 2015.

Cardona, O.D. (2004). The need for rethinking the concepts of vulnerability and risk from a holistic perspective: A necessary review and criticism for effective risk management. En: Bankoff G, Frerks G, Hilhorst D (eds.) Mapping Vulnerability: Disasters, Development and People. Earthscan Publishers, Londres. pp: $37-51$. 
CENAPRED (2014) Inundaciones. Serie: Fascículos. Centro Nacional de Prevención de Desastres. México. $56 \mathrm{p}$.

CIEDD (2014) Reporte acumulado de sismos, incendios y fenómenos hidrometeorológicos, vulnerabilidad ante inundaciones en la subcuenca Río Atoyac-Oaxaca de Juárez 2014. Oaxaca, México. Centro de Información Estadística y Documental para el Desarrollo. http://www.ciedd.oaxaca.gob.mx. Fecha de consulta el 16 de febrero de 2015.

CODICE (2010) Prediagnóstico del tema: El Agua en los Valles Centrales de Oaxaca. Centro de Acción para el Desarrollo, A.C. Oaxaca, México. 20p.

CONAPO 2010. Índice de marginación por localidad 2010. Consejo Nacional de Población. México. http://www.conapo.gob.mx/en/CONAPO/Indice_de_Marginacion_por_Localidad_2010. Fecha de consulta 8 de enero del 2016.

CONEVAL 2015. Índice de rezago social (IRS) 2015. Consejo Nacional de la Evaluación de Desarrollo Social. México. http://www.coneval.org.mx/Medicion/IRS/Paginas/Indice_Rezago_Social_2015.aspx. Fecha de consulta 8 de enero del 2016.

Cotler H (2004) El manejo integral de cuencas en México: estudios y reflexiones para orientar la política ambiental. $1^{\text {a }}$ Edición. Secretaría de Medio Ambiente y Recursos Naturales e Instituto Nacional de Ecología. México. 264p.

Cuevas ML, Garrido A, Pérez JL, González DI (2010) Estado actual de la vegetación en las cuencas de México. En: Cotler H (coord.). Las cuencas hidrográficas de México: Diagnóstico y priorización. Secretaría de Medio Ambiente y Recursos Naturales e Instituto Nacional de Ecología. México. pp: 50-59.

Chapin III FS, Matson PA, Mooney HA (2002) Principles of terrestrial ecosystem ecology. 1a Edición. Springer, New York, NY, USA. 435p.

Dang NM, Babel MS, Luong HT (2011) Evaluation of flood risk parameters in the Day river flood diversion area, Red River data, Vietnam. Natural Hazards 56: 169-194.

Dewan AM (2013) Floods in a megacity: Geospatial techniques in assessing hazards, risk and vulnerability. Springer. The Netherlands. 199p.

Eakin H, Bojórquez TL (2008) Insights into the composition of household vulnerability from multicriteria decision analysis. Global Environmental Change 18: 112-127.

Fekete A, Damm M, Birkmann J (2009) Scales as a challenge for vulnerability assessment. Natural Hazards 55: $729-747$.

Fordham M, Lovekamp EW, Thomas SKD, Phillips DB (2013) Understanding social vulnerability. En: Thomas SKD, Phillips DB, Lovekamp EW, Fothergill A (eds.) Social vulnerability to disasters. 2a Edición. CRC Press Taylor \& Francis Group. Boca Ratón, FL. USA. pp: 1-29.

Fetter CW (2001) Applied hydrogeology. 4a Edición. Prentice Hall. USA. 516p.

Gao J, Nickum JE, Pan Y (2007) An assessment of flood hazard vulnerability in the Dongting lake region of China. Lakes Reservoirs Research Management 12: 27-34.

INSO (2014) Un plan común para un bien común, hacia una estrategia articuladora de esfuerzos en pro del agua en la cuenca del Río Verde-Atoyac. Instituto de la Naturaleza y Sociedad Oaxaca. Oaxaca, México. 183p. 
Jarrett AR, Hoover JR (1985) Evaluating the effect of increasing concentrations of $\mathrm{CO}_{2}$ on infiltration rate. Transactions of the ASAE 28: 179-182.

Jha KA, Bloch RJ, Lamond J (2012) Ciudades e inundaciones: Guía para la gestión del riesgo ante inundaciones en Ciudades en el siglo 21. The World Bank-Global Facility for Disaster Reduction and Recovery. Washington, D.C. USA. 61p.

Llorente IM, Diez-Herrero A, Laín HL (2009) Aplicaciones de los SIG al análisis y gestión del riesgo de inundaciones: avances recientes. Cuadernos de la Sociedad Española de Ciencias Forestales 29: 29-37.

López MA, Sánchez L (2011) Vulnerabilidad ante inundaciones en un sector de la Ciudad de Coro sobre Sistemas de Información Geográfica. Ingeniería Hidráulica y Ambiental 32: 69-74.

Mendoza MJB, Orozco HME (2014) Análisis de la vulnerabilidad biofísica a los riesgos por inundación en la zona metropolitana de Toluca, México. Luna Azul 38: 86-104.

Meyer V, Schuer S, Haase D (2009) A multicriteria approach for flood risk mapping exemplified at the Mulde river, Germany. Natural Hazards 48: 17-39.

Montoya JV, Castillo MM, Sánchez L (2009) La importancia de las inundaciones periódicas para el funcionamiento y conservación de los ecosistemas inundables de grandes ríos tropicales: estudios en la cuenca del Orinoco. Interciencia 36: 900-907.

Palacio AA, Alfonso de APS, Casarín RS, Godínez EGB, Vanegas GP, Val SR (2005) Diagnóstico de riesgo por inundación para la ciudad de Campeche. Universidad Autónoma de Campeche, Ayuntamiento del Municipio de Campeche. Campeche, México. 109p.

PNUD (2014) Índice de desarrollo humano municipal en México: nueva metodología. Programa de las Naciones Unidas para el Desarrollo. México. 104p. http://www.mx.undp.org/dam/mexico/docs/Publica ciones/PublicacionesReduccionPobreza/InformesDesarrolloHumano/UNDP-MX-PovRed-IDHmunicipal Mexico-032014.pdf. Fecha de consulta 8 de enero del 2016.

Rodríguez ES (2011) Atlas de riesgos del municipio de Oaxaca de Juárez. Ayuntamiento de Oaxaca de Juárez-SEDESOL. Oaxaca, México. 94 p. http://www.inapam.gob.mx. Fecha de consulta 2 de mayo del 2015.

Sánchez RLA, Martínez GY (2012) Inundaciones pluviales en una cuenca urbana aplicando la ponderación mixta. Ingeniería Hidráulica y Ambiental XXXIII: 90-105.

Saaty TL (1990) Multicriteria decision making. The Analytic Hierarchy Process. Planning, priority setting, resource allocation. RWS Publications. Pittsburgh PA, USA. 287p.

Sebastian S, Haase D, Meyer V (2011) Exploring multicriteria flood vulnerability by integrating economic, social and ecological dimensions of flood risk and coping capacity: from a starting point view towards an end point view of vulnerability. Natural Hazards 58: 731-751.

Siddayo GP, Valdéz SE, Fernández LP (2014) Analytic hierarchy process (AHP) in spatial modeling for floodplain risk assessment. International Journal of Machine Learning and Computing 4: 450-457.

Torres DMC, Basurto SYYY, Cortés EJ, García UK, Koh SA, Puerto RF, Pacheco AJG (2014) Evaluación de la vulnerabilidad y el riesgo de contaminación del agua subterránea en Yucatán. Ecosistemas y Recursos Agropecuarios 1: 189-203.

Villarreal DH, Belmonte SIJ, Guevara LM (2011) Evaluación del cambio de uso de suelo en la Cuenca del Río Atoyac de Oaxaca, a través de un SIG. Memorias del II Congreso Nacional de Manejo de Cuencas Hidro- 
gráficas. Villa Hermosa, México, 18-20 de mayo. http://www.inecc.gob.mx/descargas/cuencas/2011 cnch2_dina_villareal_et_al.pdf. Fecha de consulta 10 de septiembre del 2015.

Zapata-Sierra A, Manzano-Agugliaro F (2008) Influencia de seis especies arbóreas en la infiltración de agua en el suelo. Agrociencia 42: 835-845.

Zamorano OJJ, Toscana AA (2013) El paraíso en riesgo: peligros geomorfológicos en la Bahía de Acapulco. En: Toscana AA, Monroy GJF (coord.) Riesgos y desastres. Aproximaciones teóricas y empíricas. 2a Edición. Plaza y Valdez, México. pp: 39-94. 
